
Nazeer*

\title{
Zagreb Polynomials and redefined Zagreb indices of nanostar dendrimers
}

https://doi.org/10.1515/phys-2019-0004

Received May 28, 2018; accepted January 9, 2019

\begin{abstract}
Dendrimers are profoundly extended natural macromolecules with successive layers of branch units encompassing a central core. Topological indicess are numbers related with graph of a compound to allow quantitative structureactivity/property/lethality connections. These topological indices relate certain physicochemical properties like stability, boiling point, strain energy and so forth of a compound. In this report, there have been computed redefined first, second and third Zagreb indices of Nanostar dendrimers. The authors also analyzed some Zagreb polynomials of understudy dendrimers.
\end{abstract}

Keywords: Dendrimers, Zagreb index, Zagreb polynomial, topological index, redefined Zagreb index

PACS: 81.05-t, 81.07.Nb

\section{Introduction}

Various studies have shown that there is a solid characteristic connection between synthetic properties of compounds and medications, (for example, melting point and boiling point) and their subatomic structure. The topological index characterized on the structure of these compound can enable scientists to guess physical attributes,

\footnotetext{
Shin Min Kang: Department of Mathematics and Research Institute of Natural Science, Gyeongsang National University, Jinju 52828, Korea

Center for General Education,China Medical University, Taichung 40402, Taiwan

Muhammad Yousaf: Department of Mathematics, COMSATS University Islamabad, Lahore Campus, Lahore 54000, Pakistan

Manzoor Ahmad Zahid: COMSATS University Islamabad, Sahiwal Campus, Sahiwal Pakistan

Muhammad Younas: Department of Mathematics, COMSATS University Islamabad, Lahore campus, Lahore 54000, Pakistan

*Corresponding Author: Waqas Nazeer: Division of Science and Technology, University of Education, Lahore 54000 Pakistan, E-mail: nazeer.waqas@ue.edu.pk
}

chemical reactivity and biological action $[1,2]$. In this manner, the investigation of topological indices of compounds of medications can compensate for the absence of trials and give hypothetical premise to the arrangement of medications and synthetic substances [3]. In the previous two decades, a substantial number of graph invariants (topological indices) have been considered [414] and utilized for relationship examination in chemistry, pharmacology, toxicology and ecological science. The first and second Zagreb indices [14] are one of the most established and most understood topological indices characterized by Gutman and are given distinctive names in the literature, for example, the Zagreb group index, Sag. Loeb group and the most widely recognized as Zagreb indices. The Zagreb indices are one of the primary indices acquainted and have been utilized with concentrate sub-atomic intricacy, chirality, ZE isomers and heterogeneous systems [14-17]. Dendrimers are profoundly expanded natural macromolecules with progressive layers of branch units encompassing a central core [18-21]. These are key particles in nanotechnology and can be put to great use. Nanobiotechnology is a quickly propelling region of logical and innovative opportunity that applies the instruments and procedures of nanofabrication to construct gadgets for examining biosystems. Dendrimers are one of the primary objects of this new territory of science. A dendrimer is an artificially produced or synthesized molecule developed from extended unitscalled monomers utilizing a nanoscale manufacture process. Dendrimers are perceived as one of the major commercially available nanoscale building blocks, complex and large particles with great characterized compound. From a polymer science perspective, dendrimers are almost impeccable monodisperse macromolecules with a customary and highly branched three dimensional design. They comprise of three major architectural components: core, branches and end groups. New branches emanating from a core center are included in steps until a tree-like structure is made. The nanostar dendrimer is a part of a new group of macroparticles that appear to be photon funnels just like artificial antennas.These macromolecules and all the more accurately those containing phosphorus are utilized in 
the development of nanotubes, micro and macrocapsules, nanolatex, shaded glasses, chemical sensors, modified electrodes, etc [22]. A k-polyomino framework is a limited 2-connected plane graph with the end goal that every inside face (likewise called cell) is encompassed by a normal 4k-cycle of length one. It is an edge-associated association of cells [23]. Dendrimer is a manufactured 3-dimensional macromolecule that is prepared in a step-wise fashion from straightforward branched monomer units see [24]. The nanostar dendrimer is a part of another gathering of macromolecules that appear to photon funnels simply like artificial antennas [25-28]. In this paper, the authors aim to analyze the Zagreb polynomials and redefined Zagreb indices of Nanostar dendrimers.

\section{Basic definition}

A graph is connected if there is a path between any two vertices of it. Throughout this paper $\mathrm{G}$ is a connected graph without multiple edges and loops, $V$ is the set of vertices and $\mathrm{E}$ is he set of edges. The first topological index was defined by Wiener in 1947, when he was calculating boing point of alkane [29]. Randic index is also one of the oldest topological index defined by Milan Randic [30], which has been studied extensively by both Chemists and Mathematicians due to its application in chemistry [31-35]. Gutman introduced the first and second Zagreb indices:

$$
M_{1}(G)=\sum_{u v \in E(G)}\left(d_{u}+d_{v}\right)
$$

and

$$
M_{2}(G)=\sum_{u v \in E(G)}\left(d_{u} \times d_{v}\right) .
$$

Considering the Zagreb indices, Fath-Tabar ([36-38]) defined first and the second Zagreb polynomials as

$$
M_{1}(G, x)=\sum_{u v \in E(G)} x^{d_{u}+d_{v}}
$$

and

$$
M_{2}(G, x)=\sum_{u v \in E(G)} x^{d_{u} \cdot d_{v}} .
$$

The properties of $M_{1}(G, x), M_{2}(G, x)$ polynomials for some chemical structures can be seen in the work of Gutman [39].

G. H. Fath-Tabar et al. [38] defined the third Zagreb index

$$
M_{3}(G)=\sum_{u v \in E(G)}\left(d_{u}-d_{v}\right)
$$

and the polynomial

$$
M_{3}(G, x)=\sum_{u v \in E(G)} x^{\left|d_{u}-d_{v}\right|} .
$$

In the year 2016, [40] following Zagreb type polynomials were defined

$$
\begin{aligned}
M_{4}(G, x) & =\sum_{u v \in E(G)} x^{d_{u}\left(d_{u}+d_{v}\right)} \\
M_{5}(G, x) & =\sum_{u v \in E(G)} x^{d_{v}\left(d_{u}+d_{v}\right)} \\
M_{a, b}(G, x) & =\sum_{u v \in E(G)} x^{a d_{u}+b d_{v}} \\
M_{a, b}^{\prime}(G, x) & =\sum_{u v \in E(G)} x^{\left(d_{u}+a\right)\left(d_{v}+b\right)} .
\end{aligned}
$$

Ranjini et al. [41] redefines the Zagreb index, ie, the redefined first, second and third Zagreb indices of graph $G$. These indicators appear as

$$
\begin{gathered}
\operatorname{ReZG}_{1}(G)=\sum_{u v \in E\left(P D_{1}\right)} \frac{d_{u}+d_{v}}{d_{u} \cdot d_{v}} \\
\operatorname{ReZG}_{2}(G)=\sum_{u v \in E\left(P D_{1}\right)} \frac{d_{u} \cdot d_{v}}{d_{u}+d_{v}} \\
\operatorname{ReZG}_{3}(G)=\sum_{u v \in E\left(P D_{1}\right)}\left(d_{u} \cdot d_{v}\right)\left(d_{u}+d_{v}\right) .
\end{gathered}
$$

\section{Main results}

We now consider two infinite classes $\mathrm{NS}_{1}[\mathrm{n}]$ and $\mathrm{NS}_{2}[\mathrm{n}]$ of nanostar dendrimers, see Figs. 1 and 2. Our aim is to compute the redefined Zagreb indices and Zagreb polynomials for two classes of these nanostar dendrimers. We consider the molecular graph of $\mathrm{NS}_{1}[\mathrm{n}]$ with four similar branches and three extra edges, where $n$ is steps of growth in this type of nanostars dendrimer see Figure 1.

In the following theorem we compute Zagreb polynomials of $\mathrm{NS}_{1}[\mathrm{n}]$.

Theorem 1 Let $N S_{1}[n]$ be a polypropylenimineoctaamin dendrimer, then

1.

$$
\begin{aligned}
& M_{3}\left(N S_{1}[n], x\right)=4\left(2^{n}-1\right) x^{2}+2\left(2^{n+3}-7\right) x \\
& +\left(12 \cdot 2^{n}-11\right) .
\end{aligned}
$$

2.

$$
\begin{aligned}
& M_{4}\left(N S_{1}[n], x\right)=2^{n+1} x^{3}+4\left(2^{n}-1\right) x^{4} \\
& +\left(12 \cdot 2^{n}-11\right) x^{8}+14\left(2^{n}-1\right) x^{10} .
\end{aligned}
$$




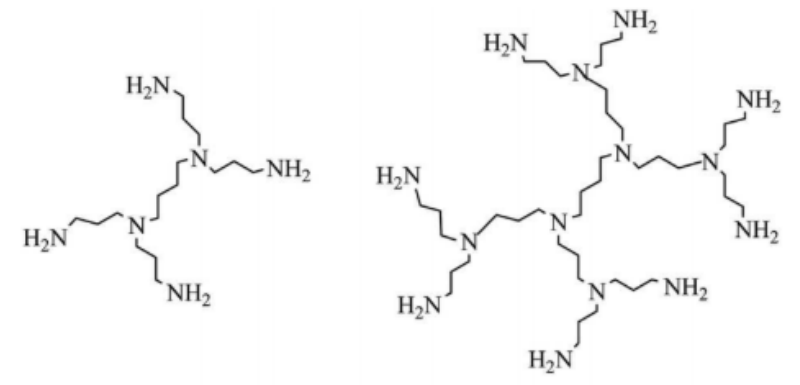

Figure 1: $N S_{1}$ [1] and $N S_{1}$ [2] polypropylenimineoctaamin dendrimer

3.

$$
\begin{aligned}
& M_{5}\left(N S_{1}[n], x\right)=2^{n+1} x^{6}+4\left(2^{n}-1\right) x^{12} \\
& +\left(12 \cdot 2^{n}-11\right) x^{8}+14\left(2^{n}-1\right) x^{15}
\end{aligned}
$$

4.

$$
\begin{aligned}
& M_{a, b}\left(N S_{1}[n], x\right)=4\left(2^{n}+2^{n-1}-1\right) x^{a} \\
& +\left(13 \cdot 2^{n+1}-25\right) x^{2 a}+\left(7 \cdot 2^{n+1}-11\right) x^{2 b} \\
& +18\left(2^{n}-1\right) x^{3 b} .
\end{aligned}
$$

5.

$$
\begin{aligned}
& M_{a, b}^{\prime}\left(N S_{1}[n], x\right)=2^{n+1} x^{(1+a)(1+b)}+4\left(2^{n}-1\right) x^{(1+a)(3+b)} \\
& +\left(12 \cdot 2^{n}-11\right) x^{(2+a)(2+b)}+14\left(2^{n}-1\right) x^{(2+a)(3+b)}
\end{aligned}
$$

Proof. From the structure of $N S_{1}[n]$, we can see that there are three partitions of vertices $V_{1}=$ $\left\{v \in V\left(N S_{1}[n]\right) \mid d_{v}=1\right\}, V_{2}=\left\{v \in V\left(N S_{1}[n]\right) \mid d_{v}=2\right\}$, and $V_{3}=\left\{v \in V\left(N S_{1}[n]\right) \mid d_{v}=3\right\}$.

The edge set of the has following four partitions:

$$
\begin{aligned}
& E_{\{1,2\}}=\left\{e=u v \in E\left(N S_{1}[n]\right) \mid d_{u}=1, d_{v}=2\right\}, \\
& E_{\{1,3\}}=\left\{e=u v \in E\left(N S_{1}[n]\right) \mid d_{u}=1, d_{v}=3\right\}, \\
& E_{\{2,2\}}=\left\{e=u v \in E\left(N S_{1}[n]\right) \mid d_{u}=2, d_{v}=2\right\}, \\
& E_{\{2,3\}}=\left\{e=u v \in E\left(N S_{1}[n]\right) \mid d_{u}=2, d_{v}=3\right\},
\end{aligned}
$$
Now

$$
\begin{gathered}
\left|E_{\{1,2\}}\right|=2^{n+1} \\
\left|E_{\{1,3\}}\right|=4\left(2^{n}-1\right) \\
\left|E_{\{2,2\}}\right|=12 \cdot 2^{n}-11 \\
\left|E_{\{2,3\}}\right|=14\left(2^{n}-1\right)
\end{gathered}
$$

1.

$$
\begin{aligned}
& M_{3}\left(N S_{1}[n], x\right)=\sum_{u v \in E\left(N S_{1}[n]\right)} x^{d_{u}-d_{v}}=\sum_{u v \in E_{\{1,2\}}\left(N S_{1}[n]\right)} x^{|2-1|} \\
& +\sum_{u v \in E_{\{1,3\}}\left(N S_{1}[n]\right)} x^{|3-1|}+\sum_{u v \in E_{\{2,2\}}\left(N S_{1}[n]\right)} x^{|2-2|} \\
& +\sum_{u v \in E_{\{2,3\}}\left(N S_{1}[n]\right)} x^{|3-2|}=\left|E_{\{1,2\}}\left(N S_{1}[n]\right)\right| x^{1} \\
& +\left|E_{\{1,3\}}\left(N S_{1}[n]\right)\right| x^{2}+\left|E_{\{2,2\}}\left(N S_{1}[n]\right)\right| x^{0} \\
& +\left|E_{\{2,3\}}\left(N S_{1}[n]\right)\right| x^{1}=4\left(2^{n}-1\right) x^{2} \\
& +2\left(2^{n+3}-7\right) x+\left(12 \cdot 2^{n}-11\right) .
\end{aligned}
$$

2.

$$
\begin{aligned}
& M_{4}\left(N S_{1}[n], x\right)=\sum_{u v \in E\left(N S_{1}[n]\right)} x^{d_{u}\left(d_{u}+d_{v}\right)}=\sum_{u v \in E_{\{1,2\}}\left(N S_{1}[n]\right)} x^{3} \\
& +\sum_{u v \in E_{\{1,3\}}\left(N S_{1}[n]\right)} x^{4}+\sum_{u v \in E_{\{2,2\}}\left(N S_{1}[n]\right)} x^{8} \\
& +\sum_{u v \in E_{\{2,3\}}\left(N S_{1}[n]\right)} x^{10}=\left|E_{\{1,2\}}\left(N S_{1}[n]\right)\right| x^{3} \\
& +\left|E_{\{1,3\}}\left(N S_{1}[n]\right)\right| x^{4}+\left|E_{\{2,2\}}\left(N S_{1}[n]\right)\right| x^{8} \\
& +\left|E_{\{2,3\}}\left(N S_{1}[n]\right)\right| x^{10}=2^{n+1} x^{3}+4\left(2^{n}-1\right) x^{4} \\
& +\left(12 \cdot 2^{n}-11\right) x^{8}+14\left(2^{n}-1\right) x^{10} .
\end{aligned}
$$$$
M_{5}\left(N S_{1}[n], x\right)=\sum_{u v \in E\left(N S_{1}[n]\right)} x^{d_{v}\left(d_{u}+d_{v}\right)}=\sum_{u v \in E_{\{1,2\}}\left(N S_{1}[n]\right)} x^{6}
$$$$
\begin{aligned}
& +\sum_{u v \in E_{\{1,3\}}\left(N S_{1}[n]\right)} x^{12}+\sum_{u v \in E_{\{2,2\}}\left(N S_{1}[n]\right)} x^{8} \\
& +\sum_{u v \in E_{\{2,3\}}\left(N S_{1}[n]\right)} x^{15}=\left|E_{\{1,2\}}\left(N S_{1}[n]\right)\right| x^{6} \\
& +\left|E_{\{1,3\}}\left(N S_{1}[n]\right)\right| x^{12}+\left|E_{\{2,2\}}\left(N S_{1}[n]\right)\right| x^{8} \\
& +\left|E_{\{2,3\}}\left(N S_{1}[n]\right)\right| x^{15}=2^{n+1} x^{6}+4\left(2^{n}-1\right) x^{12} \\
& +\left(12 \cdot 2^{n}-11\right) x^{8}+14\left(2^{n}-1\right) x^{15} .
\end{aligned}
$$

4.

$$
\begin{aligned}
& M_{a, b}\left(N S_{1}[n], x\right)=\sum_{u v \in E\left(N S_{1}[n]\right)} x^{\left(a d_{u}+b d_{v}\right)}= \\
& +\sum_{u v \in E_{\{1,2\}}\left(N S_{1}[n]\right)} x^{a+2 b}+\sum_{u v \in E_{\{1,3\}}\left(N S_{1}[n]\right)} x^{a+3 b} \\
& +\sum_{u v \in E_{\{2,2\}}\left(N S_{1}[n]\right)} x^{2 a+2 b}+\sum_{u v \in E_{\{2,3\}}\left(N S_{1}[n]\right)} x^{2 a+3 b}= \\
& +\left|E_{\{1,2\}}\left(N S_{1}[n]\right)\right| x^{a+2 b}+\left|E_{\{1,3\}}\left(N S_{1}[n]\right)\right| x^{a+3 b} \\
& 4\left(2^{n}+2^{n-1}-1\right) x^{a}+\left(13 \cdot 2^{n+1}-25\right) x^{2 a}+ \\
& \left(7 \cdot 2^{n+1}-11\right) x^{2 b}+318\left(2^{n}-1\right) x^{3 b} .
\end{aligned}
$$




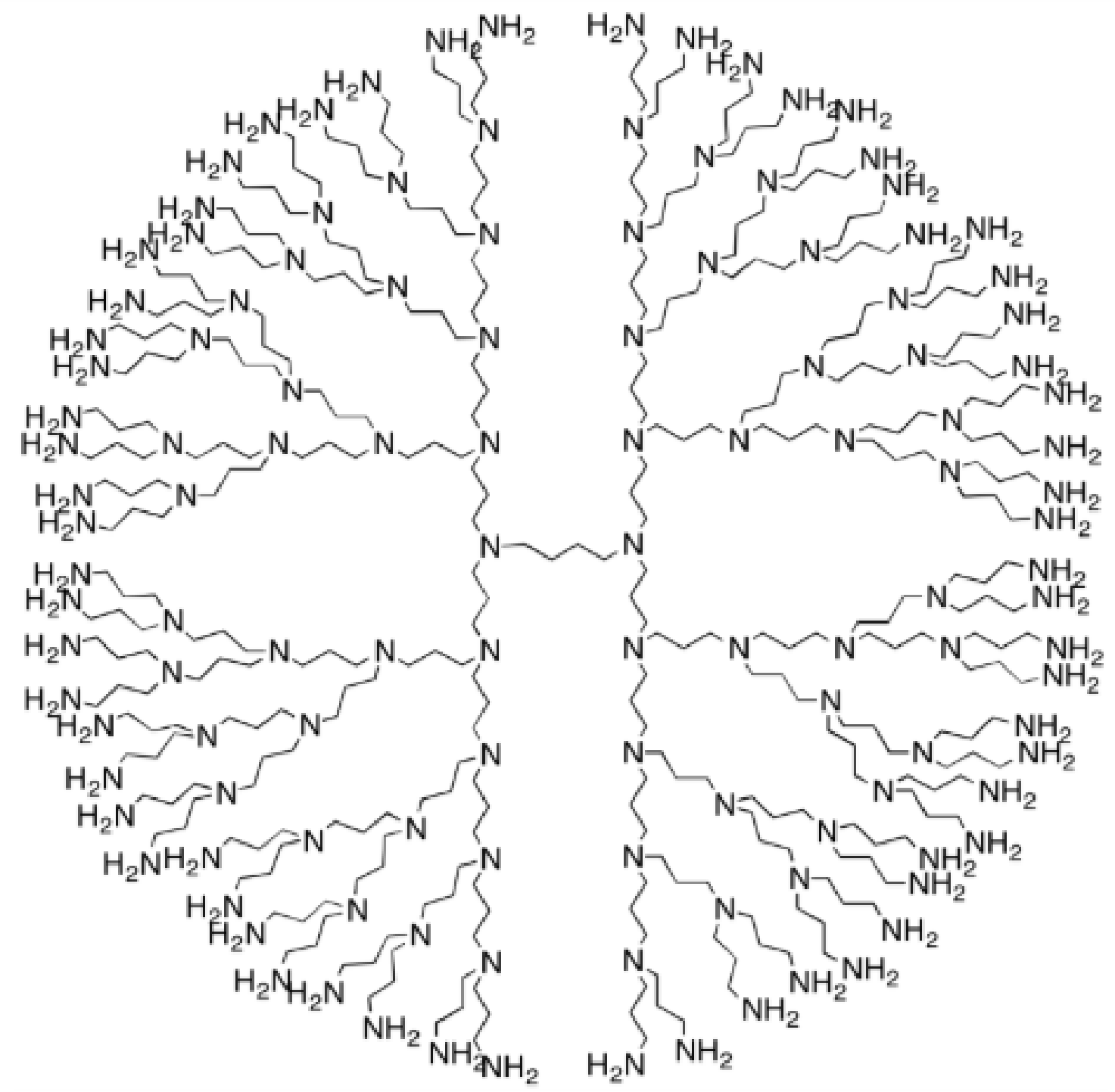

Figure 2: $N S_{2}[n]$ polypropylenimineoctaamin dendrimer

5.

$M_{a, b}^{\prime}\left(N S_{1}[n], x\right)=\sum_{u v \in E\left(N S_{1}[n]\right)} x^{\left(d_{u}+a\right)\left(d_{v}+b\right)}=$
$\sum_{u v \in E_{\{1,2\}}\left(N S_{1}[n]\right)} x^{(1+a)(2+b)}+\sum_{u v \in E_{\{1,3\}}\left(N S_{1}[n]\right)} x^{(1+a)(3+b)}$

$+\sum_{u v \in E_{\{2,2\}}\left(N S_{1}[n]\right)} x^{(2+a)(2+b)}+\sum_{u v \in E_{\{2,3\}}\left(N S_{1}[n]\right)} x^{(2+a)(3+b)}=$

$s\left|E_{\{1,2\}}\left(N S_{1}[n]\right)\right| x^{(1+a)(2+b)}+\left|E_{\{1,3\}}\left(N S_{1}[n]\right)\right| x^{(1+a)(3+b)}$

$+\left|E_{\{2,2\}}\left(N S_{1}[n]\right)\right| x^{(2+a)(2+b)}+\left|E_{\{2,3\}}\left(N S_{1}[n]\right)\right| x^{(2+a)(3+b)}$

$=2^{n+1} \chi^{(1+a)(2+b)}+4\left(2^{n}-1\right) x^{(1+a)(3+b)}$

$+\left(12 \cdot 2^{n}-11\right) x^{(2+a)(2+b)}+14\left(2^{n}-1\right) x^{(2+a)(3+b)}$.

First, second and third redefined Zagreb indices of $\mathrm{NS}_{1}[\mathrm{n}]$ has been computed in theorem 2 .
Theorem 2 Let $N S_{1}[n]$ be a polypropylenimine octaamin dendrimer, then

1.

$$
\operatorname{Re} Z G_{1}\left(N S_{1}[n]\right)=2^{n+5}-28
$$

2.

$$
\operatorname{Re} Z G_{2}\left(N S_{1}[n]\right)=\frac{2^{n+2}}{3}+\left(\frac{159}{5}\right) 2^{n}-\frac{154}{5}
$$

3. $\operatorname{Re} Z G_{3}\left(N S_{1}[n]\right)=7\left(3 \cdot 2^{n+5}-92\right)$.

Proof. From the edge partition given in theorem 2 and using definitions, we have 
1.

$$
\begin{aligned}
& \operatorname{ReZG}_{1}\left(N S_{1}[n]\right)=\sum_{u v \in E\left(N S_{1}[n]\right)} \frac{d_{u}+d_{v}}{d_{u} \cdot d_{v}} \\
& =\sum_{u v \in E_{\{1,2\}}\left(N S_{1}[n]\right)} \frac{1+2}{1 \cdot 2}+\sum_{u v \in E_{\{1,3\}}\left(N S_{1}[n]\right)} \frac{1+3}{1 \cdot 3} \\
& +\sum_{u v \in E_{\{2,2\}}\left(N S_{1}[n]\right)} \frac{2+2}{2 \cdot 2}+\sum_{u v \in E_{\{2,3\}}\left(N S_{1}[n]\right)} \frac{2+3}{2 \cdot 3} \\
& =\left|E_{\{1,2\}}\left(N S_{1}[n]\right)\right| \frac{3}{2}+\left|E_{\{1,3\}}\left(N S_{1}[n]\right)\right| \frac{4}{3} \\
& +\left|E_{\{2,2\}}\left(N S_{1}[n]\right)\right| \frac{4}{4}+\left|E_{\{2,3\}}\left(N S_{1}[n]\right)\right| \frac{5}{6}=2^{n+5}-28 .
\end{aligned}
$$

2.

$$
\begin{aligned}
& \operatorname{ReZG}_{2}\left(N S_{1}[n]\right)=\sum_{u v \in E\left(N S_{1}[n]\right)} \frac{d_{u} \cdot d_{v}}{d_{u}+d_{v}} \\
& =\sum_{u v \in E_{\{1,2\}}\left(N S_{1}[n]\right)} \frac{1 \cdot 2}{1+2}+\sum_{u v \in E_{\{1,3\}}\left(N S_{1}[n]\right)} \frac{1 \cdot 3}{1+3} \\
& +\sum_{u v \in E_{\{2,2\}}\left(N S_{1}[n]\right)} \frac{2 \cdot 2}{2+2}+\sum_{u v \in E_{\{2,3\}}\left(N S_{1}[n]\right)} \frac{2 \cdot 3}{2+3} \\
& =\left|E_{\{1,2\}}\left(N S_{1}[n]\right)\right| \frac{2}{3}+\left|E_{\{1,3\}}\left(N S_{1}[n]\right)\right| \frac{3}{4} \\
& +\left|E_{\{2,2\}}\left(N S_{1}[n]\right)\right| \frac{4}{4}+\left|E_{\{2,3\}}\left(N S_{1}[n]\right)\right| \frac{6}{5} \\
& =\frac{2^{n+2}}{3}+\left(\frac{159}{5}\right) 2^{n}-\frac{154}{5} .
\end{aligned}
$$

3.

$$
\begin{aligned}
& \operatorname{ReZG}_{3}\left(N S_{1}[n]\right)=\sum_{u v \in E\left(N S_{1}[n]\right)}\left(d_{u} \cdot d_{v}\right)\left(d_{u}+d_{v}\right) \\
& =\sum_{u v \in E_{\{1,2\}}\left(N S_{1}[n]\right)}(1 \cdot 2)(1+2) \\
& +\sum_{u v \in E_{\{1,3\}}\left(N S_{1}[n]\right)}(1 \cdot 3)(1+3) \\
& +\sum_{u v \in E_{\{2,2\}}\left(N S_{1}[n]\right)}(2 \cdot 2)(2+2) \\
& +\sum_{u v \in E_{\{2,3\}}\left(N S_{1}[n]\right)}(2 \cdot 3)(2+3)=6\left|E_{\{1,2\}}\left(N S_{1}[n]\right)\right| \\
& +12\left|E_{\{1,3\}}\left(N S_{1}[n]\right)\right|+16\left|E_{\{2,2\}}\left(N S_{1}[n]\right)\right| \\
& +30\left|E_{\{2,3\}}\left(N S_{1}[n]\right)\right|=7\left(3 \cdot 2^{n+5}-92\right) .
\end{aligned}
$$

We consider the second class of nanostar dendrimers $N S_{2}[n]$, where $n$ is steps of growth. Since the molecular graph of $G$ has four similar branches and five extra edges(see Figure 2). Next theorem is about the Zagreb polynomials of $N S_{2}[n]$.

Theorem 3 Let $N S_{2}[n]$ be the polypropylenimineoctaamin dendrimer, then
1.

$$
M_{3}\left(N S_{2}[n], x\right)=2\left(2^{n+2}-3\right) x+\left(8 \cdot 2^{n}-5\right) .
$$

2.

$$
M_{4}\left(N S_{2}[n], x\right)=2^{n+1} x^{3}+\left(8 \cdot 2^{n}-5\right) x^{8}+6\left(2^{n}-1\right) x^{10}
$$

3.

$$
M_{5}\left(N S_{2}[n], x\right)=2^{n+1} x^{6}+\left(8 \cdot 2^{n}-5\right) x^{8}+6\left(2^{n}-1\right) x^{15}
$$

4.

$$
\begin{aligned}
& M_{a, b}\left(N S_{2}[n], x\right)=2^{n+1} x^{a}+\left(7 \cdot 2^{n+1}-11\right) x^{2 a} \\
& +\left(2^{n+3}+2^{n+1}-5\right) x^{2 b}+6\left(2^{n}-1\right) x^{3 b}
\end{aligned}
$$

5.

$$
\begin{aligned}
& M_{a, b}^{\prime}\left(N S_{2}[n], x\right)=2^{n+1} \chi^{(1+a)(2+b)}+\left(8 \cdot 2^{n}-5\right) x^{(2+a)(2+b)} \\
& +6\left(2^{n}-1\right) x^{(2+a)(3+b)}
\end{aligned}
$$

Proof. From the structure of $N S_{2}[n]$, we can see that there are three partitions of vertices $V_{2}=\left\{v \in V\left(N S_{2}[n]\right) \mid d_{v}=2\right\}$, and $V_{3}=\left\{v \in V\left(N S_{2}[n]\right) \mid d_{v}=3\right\}$,

The edge set of the $N S_{2}[n]$ has following four partitions;

$$
\begin{aligned}
& E_{\{1,2\}}=\left\{e=u v \in E\left(N S_{2}[n]\right) \mid d_{u}=1, d_{v}=2\right\}, \\
& E_{\{2,2\}}=\left\{e=u v \in E\left(N S_{2}[n]\right) \mid d_{u}=2, d_{v}=2\right\}, \\
& E_{\{2,3\}}=\left\{e=u v \in E\left(N S_{2}[n]\right) \mid d_{u}=2, d_{v}=3\right\},
\end{aligned}
$$

Now

$$
\begin{gathered}
\left|E_{\{1,2\}}\right|=2^{n+1} \\
\left|E_{\{2,2\}}\right|=8 \cdot 2^{n}-5
\end{gathered}
$$

1.

$$
\begin{aligned}
& M_{3}\left(N S_{2}[n], x\right)=\sum_{u v \in E\left(N S_{2}[n]\right)} x^{\left|d_{u}-d_{v}\right|}=\sum_{u v \in E_{\{1,2\}}\left(N S_{2}[n]\right)} x^{|2-1|} \\
& +\sum_{u v \in E_{\{2,2\}}\left(N S_{2}[n]\right)} x^{|2-2|}+\sum_{u v \in E_{\{2,3\}}\left(N S_{2}[n]\right)} \\
& =\left|E_{\{1,2\}}\left(N S_{2}[n]\right)\right| x^{1} \\
& +\left|E_{\{2,2\}}\left(N S_{2}[n]\right)\right| x^{0}+\left|E_{\{2,3\}}\left(N S_{2}[n]\right)\right| x^{1} \\
& =2\left(2^{n+2}-3\right) x+\left(8 \cdot 2^{n}-5\right) .
\end{aligned}
$$


2.

$$
\begin{aligned}
& M_{4}\left(N S_{2}[n], x\right)=\sum_{u v \in E\left(N S_{2}[n]\right)} x^{d_{u}\left(d_{u}+d_{v}\right)}=\sum_{u v \in E_{\{1,2\}}\left(N S_{2}[n]\right)} x^{3} \\
& +\sum_{u v \in E_{\{2,2\}}\left(N S_{2}[n]\right)} x^{8}+\sum_{u v \in E_{\{2,3\}}\left(N S_{2}[n]\right)} x^{10} \\
& =\left|E_{\{1,2\}}\left(N S_{2}[n]\right)\right| x^{3}+\left|E_{\{2,2\}}\left(N S_{2}[n]\right)\right| x^{8} \\
& +\left|E_{\{2,3\}}\left(N S_{2}[n]\right)\right| x^{10}=2^{n+1} x^{3}+\left(8 \cdot 2^{n}-5\right) x^{8} \\
& +6\left(2^{n}-1\right) x^{10} .
\end{aligned}
$$

3.

$$
\begin{aligned}
& M_{5}\left(N S_{2}[n], x\right)=\sum_{u v \in E\left(N S_{2}[n]\right)} x^{d_{v}\left(d_{u}+d_{v}\right)}=\sum_{u v \in E_{\{1,2\}}\left(N S_{2}[n]\right)} x^{6} \\
& +\sum_{u v \in E_{\{2,2\}}\left(N S_{2}[n]\right)} x^{8}+\sum_{u v \in E_{\{2,3\}}\left(N S_{2}[n]\right)} x^{15} \\
& =\left|E_{\{1,2\}}\left(N S_{2}[n]\right)\right| x^{6}+\left|E_{\{2,2\}}\left(N S_{2}[n]\right)\right| x^{8} \\
& +\left|E_{\{2,3\}}\left(N S_{2}[n]\right)\right| x^{15}=2^{n+1} x^{6}+\left(8 \cdot 2^{n}-5\right) x^{8} \\
& +6\left(2^{n}-1\right) x^{15} .
\end{aligned}
$$

4.

$$
\begin{aligned}
& M_{a, b}\left(N S_{2}[n], x\right)=\sum_{u v \in E\left(N S_{2}[n]\right)} x^{\left(a d_{u}+b d_{v}\right)} \\
& =\sum_{u v \in E_{\{1,2\}}\left(N S_{2}[n]\right)} x^{a+2 b}+\sum_{u v \in E_{\{2,2\}}\left(N S_{2}[n]\right)} x^{2 a+2 b} \\
& +\sum_{u v \in E_{\{2,3\}}\left(N S_{2}[n]\right)} x^{2 a+3 b}=\left|E_{\{1,2\}}\left(N S_{2}[n]\right)\right| x^{a+2 b} \\
& +\left|E_{\{2,2\}}\left(N S_{2}[n]\right)\right| x^{2 a+2 b}+\left|E_{\{2,3\}}\left(N S_{2}[n]\right)\right| x^{2 a+3 b} \\
& =2^{n+1} x^{a}+\left(7 \cdot 2^{n+1}-11\right) x^{2 a}+\left(2^{n+3}+2^{n+1}-5\right) x^{2 b} \\
& +6\left(2^{n}-1\right) x^{3 b} .
\end{aligned}
$$

5.

$$
\begin{aligned}
& M_{a, b}^{\prime}\left(N S_{2}[n], x\right)=\sum_{u v \in E\left(N S_{2}[n]\right)} x^{\left(d_{u}+a\right)\left(d_{v}+b\right)} \\
& =\sum_{u v \in E_{\{1,2\}}\left(N S_{2}[n]\right)} x^{(1+a)(2+b)}+\sum_{u v \in E_{\{2,2\}}\left(N S_{2}[n]\right)} x^{(2+a)(2+b)} \\
& +\sum_{u v \in E_{\{2,3\}}\left(N S_{2}[n]\right)} x^{(2+a)(3+b)}=\left|E_{\{1,2\}}\left(N S_{2}[n]\right)\right| x^{(1+a)(2+b)} \\
& +\left|E_{\{2,2\}}\left(N S_{2}[n]\right)\right| x^{(2+a)(2+b)}+\left|E_{\{2,3\}}\left(N S_{2}[n]\right)\right| x^{(2+a)(3+b)} \\
& =2^{n+1} x^{(1+a)(2+b)}+\left(8 \cdot 2^{n}-5\right) x^{(2+a)(2+b)} \\
& +6\left(2^{n}-1\right) x^{(2+a)(3+b)} .
\end{aligned}
$$

Next theorem is about the first, second and third redefined Zagreb indices.

Theorem 4 Let $N S_{2}[n]$ be the polypropylenimine octaamin dendrimer, then
1.

2.

$$
\operatorname{Re} Z G_{2}\left(N S_{2}[n]\right)=\left(\frac{31}{15}\right) 2^{n+3}-\frac{61}{5} .
$$

3.

$$
\operatorname{Re} Z G_{3}\left(N S_{2}[n]\right)=5 \cdot 2^{n+6}-260 .
$$

Proof. From the edge partition given in theorem 3 and using definitions, we have 1.

$$
\begin{aligned}
& \operatorname{ReZG}_{1}\left(N S_{2}[n]\right)=\sum_{u v \in E\left(N S_{2}[n]\right)} \frac{d_{u}+d_{v}}{d_{u} \cdot d_{v}} \\
& =\sum_{u v \in E_{\{1,2\}}\left(N S_{2}[n]\right)} \frac{1+2}{1 \cdot 2}+\sum_{u v \in E_{\{2,2\}}\left(N S_{2}[n]\right)} \frac{2+2}{2 \cdot 2} \\
& +\sum_{u v \in E_{\{2,3\}}\left(N S_{2}[n]\right)} \frac{2+3}{2 \cdot 3}=\left|E_{\{1,2\}}\left(N S_{2}[n]\right)\right| \frac{3}{2} \\
& +\left|E_{\{1,3\}}\left(N S_{2}[n]\right)\right|+\left|E_{\{2,2\}}\left(N S_{2}[n]\right)\right| \frac{5}{6}=2^{n+4}-10 .
\end{aligned}
$$

2.

$$
\begin{aligned}
& \operatorname{ReZG}_{2}\left(N S_{2}[n]\right)=\sum_{u v \in E\left(N S_{2}[n]\right)} \frac{d_{u} \cdot d_{v}}{d_{u}+d_{v}} \\
& =\sum_{u v \in E_{\{1,2\}}\left(N S_{2}[n]\right)} \frac{1 \cdot 2}{1+2}+\sum_{u v \in E_{\{2,2\}}\left(N S_{2}[n]\right)} \frac{2 \cdot 2}{2+2} \\
& +\sum_{u v \in E_{\{2,3\}}\left(N S_{2}[n]\right)} \frac{2 \cdot 3}{2+3}=\left|E_{\{1,2\}}\left(N S_{1}[n]\right)\right| \frac{2}{3} \\
& +\left|E_{\{2,2\}}\left(N S_{2}[n]\right)\right|+\left|E_{\{2,3\}}\left(N S_{2}[n]\right)\right| \frac{6}{5} \\
& =\left(\frac{31}{15}\right) 2^{n+3}-\frac{61}{5} .
\end{aligned}
$$

3.

$$
\begin{aligned}
& \operatorname{ReZG}_{3}\left(N S_{2}[n]\right)=\sum_{u v \in E\left(N S_{2}[n]\right)}\left(d_{u} \cdot d_{v}\right)\left(d_{u}+d_{v}\right) \\
& =\sum_{u v \in E_{\{1,2\}}\left(N S_{2}[n]\right)}(1 \cdot 2)(1+2) \\
& +\sum_{u v \in E_{\{2,2\}}\left(N S_{2}[n]\right)}(2 \cdot 2)(2+2) \\
& +\sum_{u v \in E_{\{2,3\}}\left(N S_{2}[n]\right)}(2 \cdot 3)(2+3)=6\left|E_{\{1,2\}}\left(N S_{2}[n]\right)\right| \\
& +16\left|E_{\{2,2\}}\left(N S_{2}[n]\right)\right|+30\left|E_{\{2,3\}}\left(N S_{2}[n]\right)\right| \\
& =5 \cdot 2^{n+6}-260 .
\end{aligned}
$$

Next, we compute Zagreb polynomials and redefined Zagreb indices of $D[n]$ shown in Figures 3 and 4. 


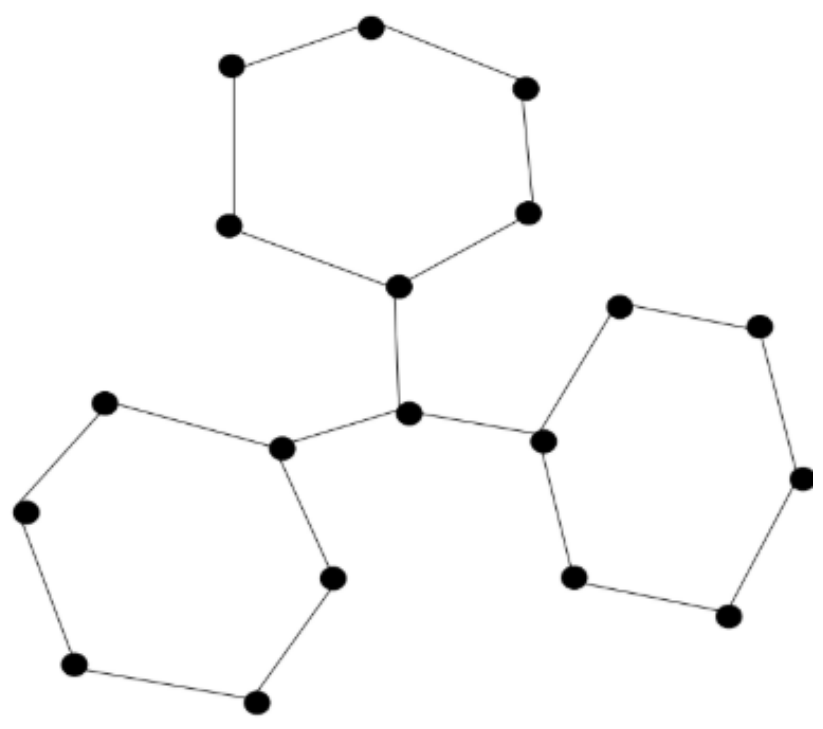

Figure 3: $D[1]$ Nanostar dendrimer

Theorem 5 Let $D_{n}$ be the nanostar dendrimer, then

1.

$$
M_{3}\left(D_{n}, x\right)=6\left(5 \cdot 2^{n-1}-4\right) x+3\left(3 \cdot 2^{n+1}-7\right)
$$

2.

$$
\begin{aligned}
& M_{5}\left(D_{n}, x\right)=12\left(2^{n}-1\right) x^{8}+6\left(5 \cdot 2^{n-1}-4\right) x^{15} \\
& +3\left(2^{n+1}-3\right) x^{18}
\end{aligned}
$$

3.

$$
\begin{aligned}
& M_{a, b}\left(D_{n}, x\right)=9\left(3 \cdot 2^{n}-4\right) x^{2 a}+12\left(2^{n}-1\right) x^{2 b} \\
& +3\left(2^{n+1}-3\right) x^{3 a}+3\left(7 \cdot 2^{n}-11\right) x^{3 b}
\end{aligned}
$$

4.

$$
\begin{aligned}
& M_{a, b}^{\prime}\left(D_{n}, x\right)=12\left(2^{n}-1\right) x^{(2+a)(2+b)} \\
& +6\left(5 \cdot 2^{n-1}-4\right) x^{(2+a)(3+b)}+3\left(2^{n+1}-3\right) x^{(3+a)(3+b)} .
\end{aligned}
$$

Proof. From the structure of $D_{n}$, we can see that there are three partitions of vertices

and

$$
V_{2}=\left\{v \in V\left(D_{n}\right) \mid d_{v}=2\right\},
$$

$V_{3}=\left\{v \in V\left(D_{n}\right) \mid d_{v}=3\right\}$,

The edge set of the $D_{n}$ has following four partitions;

$$
\begin{aligned}
& E_{\{1,2\}}=\left\{e=u v \in E\left(D_{n}\right) \mid d_{u}=2, d_{v}=2\right\}, \\
& E_{\{2,2\}}=\left\{e=u v \in E\left(D_{n}\right) \mid d_{u}=2, d_{v}=3\right\}, \\
& E_{\{2,3\}}=\left\{e=u v \in E\left(D_{n}\right) \mid d_{u}=3, d_{v}=3\right\},
\end{aligned}
$$

Now

$$
\begin{gathered}
\left|E_{\{1,2\}}\right|=12\left(2 \cdot 2^{n-1}-1\right) \\
\left|E_{\{2,3\}}\right|=12 \cdot 2^{n-1}-9
\end{gathered}
$$

1.

$$
\begin{aligned}
& M_{3}\left(\left(D_{n}\right), x\right)=\sum_{u v \in E\left(D_{n}\right)} x^{\left|d_{u}-d_{v}\right|}=\sum_{u v \in E_{\{1,2\}}\left(D_{n}\right)} x^{|2-1|} \\
& +\sum_{u v \in E_{\{2,2\}}\left(D_{n}\right)} x^{|2-2|}+\sum_{u v \in E_{\{2,3\}}\left(D_{n}\right)} x^{|2-3|} \\
& =\left|E_{\{1,2\}}\left(D_{n}\right)\right| x^{1}+\left|E_{\{2,2\}}\left(D_{n}\right)\right| x^{0} \\
& +\left|E_{\{2,3\}}\left(D_{n}\right)\right| x^{1}=6\left(5 \cdot 2^{n-1}-4\right) x \\
& +3\left(3 \cdot 2^{n+1}-7\right) .
\end{aligned}
$$

2.

$$
\begin{aligned}
& M_{4}\left(\left(D_{n}\right), x\right)=\sum_{u v \in E\left(D_{n}\right)} x^{d_{u}\left(d_{u}+d_{v}\right)}=\sum_{u v \in E_{\{1,2\}}\left(D_{n}\right)} x^{3} \\
& +\sum_{u v \in E_{\{2,2\}}\left(D_{n}\right)} x^{8}+\sum_{u v \in E_{\{2,3\}}\left(D_{n}\right)} x^{10}=\left|E_{\{1,2\}}\left(D_{n}\right)\right| x^{3} \\
& +\left|E_{\{2,2\}}\left(D_{n}\right)\right| x^{8}+\left|E_{\{2,3\}}\left(D_{n}\right)\right| x^{10} \\
& =12\left(2^{n}-1\right) x^{8}+\left(5 \cdot 2^{n-1}-4\right) x^{10} \\
& +3\left(2^{n+1}-3\right) x^{18}
\end{aligned}
$$

3.

$$
\begin{aligned}
& M_{5}\left(\left(D_{n}\right), x\right)=\sum_{u v \in E\left(D_{n}\right)} x^{d_{v}\left(d_{u}+d_{v}\right)}=\sum_{u v \in E_{\{1,2\}}\left(D_{n}\right)} x^{6} \\
& +\sum_{u v \in E_{\{2,2\}}\left(D_{n}\right)} x^{8}+\sum_{u v \in E_{\{2,3\}}\left(D_{n}\right)} x^{15}=\left|E_{\{1,2\}}\left(D_{n}\right)\right| x^{6} \\
& +\left|E_{\{2,2\}}\left(D_{n}\right)\right| x^{8}+\left|E_{\{2,3\}}\left(D_{n}\right)\right| x^{15}=12\left(2^{n}-1\right) x^{8} \\
& +6\left(5 \cdot 2^{n-1}-4\right) x^{15}+3\left(2^{n+1}-3\right) x^{18} .
\end{aligned}
$$

4.

$$
\begin{aligned}
& M_{a, b}\left(\left(D_{n}\right), x\right)=\sum_{u v \in E\left(D_{n}\right)} x^{\left(a d_{u}+b d_{v}\right)}=\sum_{u v \in E_{\{1,2\}}\left(D_{n}\right)} x^{a+2 b} \\
& +\sum_{u v \in E_{\{2,2\}}\left(D_{n}\right)} x^{2 a+2 b}+\sum_{u v \in E_{\{2,3\}}\left(D_{n}\right)} x^{2 a+3 b} \\
& =\left|E_{\{1,2\}}\left(D_{n}\right)\right| x^{a+2 b}+\left|E_{\{2,2\}}\left(D_{n}\right)\right| x^{2 a+2 b} \\
& +\left|E_{\{2,3\}}\left(D_{n}\right)\right| x^{2 a+3 b}=9\left(3 \cdot 2^{n}-4\right) x^{2 a} \\
& +12\left(2^{n}-1\right) x^{2 b}+3\left(2^{n+1}-3\right) x^{3 a} \\
& +3\left(7 \cdot 2^{n}-11\right) x^{3 b} .
\end{aligned}
$$




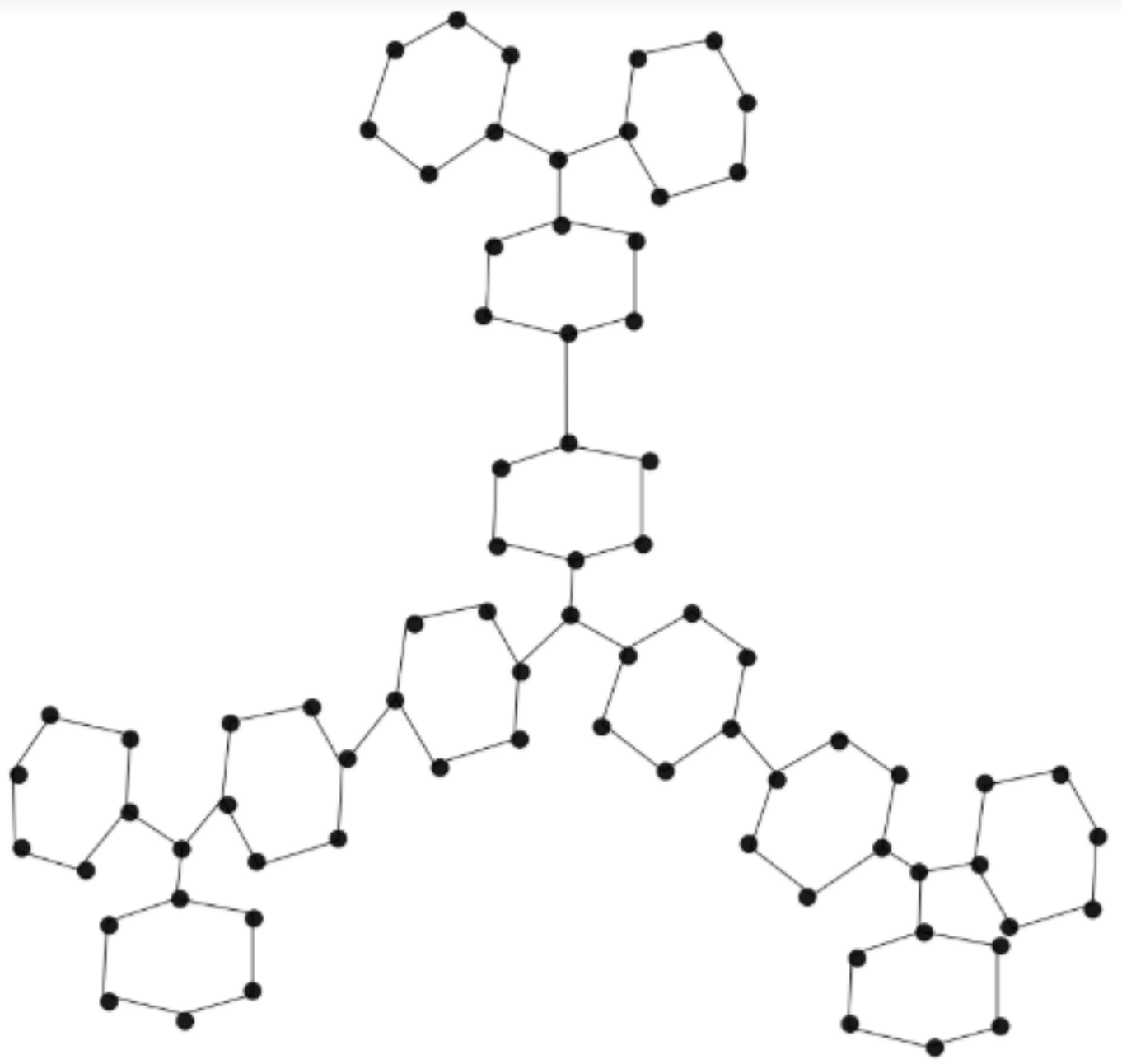

Figure 4: $D[2]$ Nanostar dendrimer

5.

$$
\begin{aligned}
& \left.M_{a, b}^{\prime}\left(D_{n}\right), x\right)=\sum_{u v \in E\left(D_{n}\right)} x^{\left(d_{u}+a\right)\left(d_{v}+b\right)} \\
& =\sum_{u v \in E_{\{1,2\}}\left(D_{n}\right)} x^{(1+a)(2+b)}+\sum_{u v \in E_{\{2,2\}}\left(D_{n}\right)} x^{(2+a)(2+b)} \\
& +\sum_{u v \in E_{\{2,3\}}\left(D_{n}\right)} x^{(2+a)(3+b)}=\left|E_{\{1,2\}}\left(D_{n}\right)\right| x^{(1+a)(2+b)} \\
& +\left|E_{\{2,2\}}\left(D_{n}\right)\right| x^{(2+a)(2+b)}+\left|E_{\{2,3\}}\left(D_{n}\right)\right| x^{(2+a)(3+b)} \\
& =12\left(2^{n}-1\right) x^{(2+a)(2+b)}+6\left(5 \cdot 2^{n-1}-4\right) x^{(2+a)(3+b)} \\
& +3\left(2^{n+1}-3\right) x^{(3+a)(3+b)} .
\end{aligned}
$$

2. $\operatorname{Re} Z G_{3}\left(D_{n}\right)=3 \cdot 2^{n+6}+387 \cdot 2^{n+1}-1398$.

Proof. From the edge partition given in theorem 5 and using definitions, we have

1.

$$
\begin{aligned}
& \operatorname{ReZG}_{1}\left(D_{n}\right)=\sum_{u v \in E\left(D_{n}\right)} \frac{d_{u}+d_{v}}{d_{u} \cdot d_{v}}=\sum_{u v \in E_{\{1,2\}}\left(D_{n}\right)} \frac{1+2}{1 \cdot 2} \\
& +\sum_{u v \in E_{\{2,2\}}\left(D_{n}\right)} \frac{2+2}{2 \cdot 2}+\sum_{u v \in E_{\{2,3\}}\left(D_{n}\right)} \frac{2+3}{2 \cdot 3} \\
& =\left|E_{\{1,2\}}\left(D_{n}\right)\right| \frac{3}{2}+\left|E_{\{1,3\}}\left(D_{n}\right)\right|+\left|E_{\{2,2\}}\left(D_{n}\right)\right| \frac{5}{6} \\
& =3 \cdot 2^{n+2}+33 \cdot 2^{n-1}-38 .
\end{aligned}
$$

Theorem 6 Let $D_{n}$ be the nanostar dendrimer, then

1.

$$
\operatorname{Re} Z G_{2}\left(D_{n}\right)=3 \cdot 2^{n+2}+27 \cdot 2^{n}-\frac{423}{10} \text {. }
$$


2.

$$
\begin{aligned}
& \operatorname{ReZG}_{2}\left(D_{n}\right)=\sum_{u v \in E\left(D_{n}\right)} \frac{d_{u} \cdot d_{v}}{d_{u}+d_{v}}=\sum_{u v \in E_{\{1,2\}}\left(D_{n}\right)} \frac{1 \cdot 2}{1+2} \\
& +\sum_{u v \in E_{\{2,2\}}\left(D_{n}\right)} \frac{2 \cdot 2}{2+2}+\sum_{u v \in E_{\{2,3\}}\left(D_{n}\right)} \frac{2 \cdot 3}{2+3} \\
& =\left|E_{\{1,2\}}\left(D_{n}\right)\right| \frac{2}{3}+\left|E_{\{2,2\}}\left(D_{n}\right)\right|+\left|E_{\{2,3\}}\left(D_{n}\right)\right| \frac{6}{5} \\
& =3 \cdot 2^{n+2}+27 \cdot 2^{n}-\frac{423}{10} .
\end{aligned}
$$

\section{Conclusion}

QSARs speak to prescient models got from utilization of measurable devices connecting natural action (counting attractive remedial impact and bothersome reactions) of synthetic activities (drugs/toxicants/ecological contaminations) with descriptors illustrative of atomic structure as well as properties. QSARs are being connected in numerous orders for instance hazard evaluation, lethality expectation, and administrative choices notwithstanding drug revelation and lead improvement. For instance, ETA index have been connected in the advancement of prescient QSAR/QSPR/QSTR models. Dendrimers have lot of application in biology [42], drugs [43], chemistry [44], etc. In this paper, the authors computed the first, second and third redefined Zagreb indices of nanostar dendrimersand $D(n)$. The authors also analyzed Zagreb polynomials of understudy nanostar dendrimers. Topological indices computed in this paper can help to understand the structure of dendrimers and with the help of these topological indices we can guess many physicochemical properties of Nanostar dendrimers. See [45-47] for more application.

Acknowledgement: The authors are thankful to both the reviewers for valuable comments that improve the quality of this paper.

\section{References}

[1] James D., Balaban A.T., eds. Topological indices and related descriptors in QSAR and QSPAR. CRC Press, 2000.

[2] Balaban A.T., Motoc I., Bonchev D., Mekenyan O., Topological indices for structure-activity correlations, In: Steric effects in drug design, 1983, Springer, Berlin, Heidelberg.

[3] Estrada E., Uriarte E., Recent advances on the role of topological indices in drug discovery research, Curr. Med. Chem., 2001, 8(13), 1573-1588.
[4] Alaeiyan M., Farahani M.R., Jamil M.K., Computation of the fifth geometric-arithmetic index for polycyclic aromatic hydrocarbons PaHk, Appl. Math. Nonlin. Sci., 2016, 1(1), 283-290.

[5] Jamil M.K., Farahani M.R., Imran M., Malik M.A., Computing eccentric version of second zagreb index of polycyclic aromatic hydrocarbons PAHk, Appl. Math. Nonlin. Sci., 2016, 1(1), 247-252.

[6] Farahani M.R., Jamil M.K., Imran M., Vertex Plv topological index of titania carbon nanotubes TiO2 (m, n), Appl. Math. Nonl. Sc, 2016, 1(1), 175-182.

[7] Gao W., Zali M.R., Degree-based indices computation for special chemical molecular structures using edge dividing method, Appl. Math. Nonlin. Sci., 2016, 1(1), 94-117.

[8] Basavanagoud B., Gao W., Patil S., Desai V.R., Mirajkar K.G., Balani P., Computing First Zagreb index and F-index of New Cproducts of Graphs, Appl. Math. Nonlin. Sci., 2017, 2(1), 285298.

[9] Lokesha V., Deepika T., Ranjini P.S., Cangul I.N., Operations of Nanostructures via SDD, ABC4 and GA5 indices, Appl. Math. Nonlin. Sci., 2017, 2(1), 173-180.

[10] Hosamani S.M., Kulkarni B.B., Boli R.G., Gadag V.M., QSPR analysis of certain graph theocratical matrices and their corresponding energy, Appl. Math. Nonlin. Sci., 2017, 2(1), 131-150.

[11] Sardar M.S., Zafar S., Zahid Z., Computing topological indices of the line graphs of Banana tree graph and Firecracker graph, Appl. Math. Nonlin. Sci., 2017, 2(1), 83-92.

[12] Basavanagoud B., Desai V.R., Patil S., $(\beta, \alpha)$ - Connectivity Index of Graphs, Applied Mathematics and Nonlinear Sciences, 2017, 2(1), 21-30.

[13] Ramane H.S., Jummannaver R.B., Note on forgotten topological index of chemical structure in drugs, Appl. Math. Nonlin. Sci., 2016,1(2), 369-374.

[14] Gutman I., Das K. C., The first Zagreb index 30 years after, MATCH Commun. Math. Comput. Chem, 2004, 50(1), 83-92.

[15] Miličević A., Nikolić S., On variable Zagreb indices Croatica chemica acta, 2004, 77(1-2), 97-101.

[16] Bonche D., Trinajstič N., Overall molecular descriptors. 3. Overall Zagreb indices, SAR and QSAR in Environmental Research, 2001, 12(1-2), 213-236.

[17] Bajaj S., Sambi S S., Madan A.K., Prediction of antiinflammatory activity of $\mathrm{N}$-arylanthranilic acids: computational approach using refined Zagreb indices, Croat Chem Acta, 2005, 78(2), 165-174.

[18] Borovicanin B., On the extremal Zagreb indices of trees with given number of segments or given number of branching vertices, MATCH Commun. Math. Comput. Chem, 2015, 74(1), 57-79.

[19] Chen Z., Dehmer M., Emmert-Streib F., Shi Y., Entropy bounds for dendrimers, Appl. Math. Comput., 2014, 242, 462-472.

[20] Siddiqui M.K., Imran M., Ahmad A., On Zagreb indices, Zagreb polynomials of some nanostar dendrimers, Applied Mathematics and Computation, 2016, 280, 132-139.

[21] Barbara K., Maria B., Review Dendrimers: properties and applications, Acta Biochim. Pol., 2001, 48(1), 199-208.

[22] De N., Nayeem S M.A., Computing the F-index of nanostar dendrimers, Pacific Sci. Rev. A: Nat. Sci. Eng., 2016, 18(1), 14-21.

[23] Toth C.D., O'Rourke J., Goodman J.E., Handbook of discrete and computational geometry, Chapman and Hall/CRC 2017.

[24] Naylor A.M., Goddard W.A., Kiefer G.E., Tomalia D.A., Starburst dendrimers. 5. Molecular shape control, J. Amer. Chem. Soc., 1989, 111(6), 2339-2341. 
[25] Ghorbani M., Hosseinzadeh M.A., Computing $A B C 4$ index of nanostar dendrimers, Optoelectron. Adv. Mater. Rapid Commun., 2010, 4(9), 1419-1422.

[26] Karbasioun A., Ashrafi A.R., Wiener and detour indices of a new type of nanostar dendrimers, Macedonian J. Chem. Chem. Eng., 2009, 28(1), 49-54.

[27] Ashrafi A.R., Nikzad P.,Keküle index and bounds of energy for nanostar dendrimers, Digest J. Nanomat. Biostr. (DJNB), 2009, 4(2).

[28] Munir M., Nazeer W., Rafique S., Kang S.M., M-polynomial and related topological indices of Nanostar dendrimers, Symmetry, 2016, 8(9), 97.

[29] Wiener H., Structural determination of paraffin boiling points, J. Amer. Chem. Soc., 1947, 69(1), 17-20.

[30] Randic M., Characterization of molecular branching, J. Amer. Chem. Soc., 1975, 97(23), 6609-6615.

[31] Li X., Shi Y., A survey on the Randic index, MATCH Commun. Math. Comput. Chem, 2008, 59(1), 127-156.

[32] Hu Y., Li X., Shi Y., Xu T., Gutman I., On molecular graphs with smallest and greatest zeroth-order general Randic index, MATCH Commun. Math. Comput. Chem, 2005, 54(2), 425-434.

[33] Cavers M., Fallat S., Kirkland S., On the normalized Laplacian energy and general Randic index R-1 of graphs, Linear Algebra Appl., 2010, 433(1), 172-190.

[34] Li X., Yang Y., Sharp bounds for the general Randic index, MATCH Commun. Math. Comput. Chem, 2004, 51, 155-166.

[35] Hu Y., Li X., Yuan Y., Trees with minimum general Randic index, MATCH Commun. Math. Comput. Chem, 2004, 52, 119-128.

[36] Aouchiche M., Hansen P., On a conjecture about the Randić index, Discr. Math., 2007, 307(2), 262-265.

[37] Fath-Tabar G.H., Old and new Zagreb index, MATCH-Commun. Math. Comput. Chem., 2011, 65, 79-84.
[38] Astaneh-Asl A., Fath-Tabar G. H., Computing the first and third Zagreb polynomials of Cartesian product of graphs, Iranian J. Math. Chem., 2011, 2(2), 73-78.

[39] Fath-Tabar G.H., Zagreb Polynomial and Pi Indices of some Nano Structures, Digest J. Nanomat. Biostr., 2009, 4(1), 189-191.

[40] Gutman I., New bounds on zagreb indices and the zagreb coindices, Bol. Soc. Paran Mat. 2013, 31(1), 51-65.

[41] Bindusree A.R., Cangul I.N., Lokesha V., Cevik A.S., Zagreb polynomials of three graph operators, Filomat., 2016, 30(7), 19791986.

[42] Ranjini P.S., Lokesha V., Usha A., Relation between phenylene and hexagonal squeeze using harmonic index, Int. J. Graph Theory, 2013, 1, 116-121

[43] Lee C.C., MacKay J.A., Fréchet J.M., Szoka F.C., Designing dendrimers for biological applications, Nature Biotechnol., 2005, 23(12), 1517.

[44] Esfand R., Tomalia D.A., Poly (amidoamine)(PAMAM) dendrimers: from biomimicry to drug delivery and biomedical applications, Drug Discovery Today, 2001, 6(8), 427-436.

[45] Bosko J.T., Prakash, R.J., Effect of molecular topology on the transport properties of dendrimers in dilute solution at $\theta$ temperature: A Brownian dynamics study, J. Chem. Phys., 2008, 128(3), 034902.

[46] Shuxian L., Zagreb polynomials of thorn graphs, Kragujevac J. Sci., 2011, 33, 33-38.

[47] Guariglia E., Silvestrov S., Fractional-Wavelet Analysis of Positive definite Distributions and Wavelets on D'(C), in Engineering Mathematics II, Silvestrov, Rancic (Eds.), 2016, 337-353, Springer.

[48] Guariglia E., Spectral Analysis of the Weierstrass-Mandelbrot Function, In: Proceeding of the 2nd International Multidisciplinary Conference on Computer and Energy Science, Split, Croatia, 12-14 July 2017. 